\title{
Raman Spectroscopy of DLC/a-Si Bilayer Film Prepared by Pulsed Filtered Cathodic Arc
}

\author{
C. Srisang, ${ }^{1,2,3}$ P. Asanithi, ${ }^{1}$ K. Siangchaew, ${ }^{2}$ S. Limsuwan, ${ }^{1}$ \\ A. Pokaipisit, ${ }^{1,3}$ and P. Limsuwan ${ }^{1,3}$ \\ ${ }^{1}$ Department of Physics, Faculty of Science, King Mongkut's University of Technology Thonburi, Bangkok 10140, Thailand \\ ${ }^{2}$ Western Digital (Thailand) Company Limited, Ayuthaya 13160, Thailand \\ ${ }^{3}$ Thailand Center of Excellence in Physics, CHE, Ministry of Education, Bangkok 10400, Thailand
}

Correspondence should be addressed to A. Pokaipisit, pokaipisit@gmail.com

Received 1 August 2012; Revised 4 October 2012; Accepted 4 October 2012

Academic Editor: Sheng-Rui Jian

Copyright () 2012 C. Srisang et al. This is an open access article distributed under the Creative Commons Attribution License, which permits unrestricted use, distribution, and reproduction in any medium, provided the original work is properly cited.

\begin{abstract}
DLC/a-Si bilayer film was deposited on germanium substrate. The a-Si layer, a seed layer, was firstly deposited on the substrate using DC magnetron sputtering and DLC layer was then deposited on the a-Si layer using pulsed filtered cathodic arc method. The bilayer films were deposited with different DLC/a-Si thickness ratios, including 2/2, 2/6, 4/4, 6/2, and 9/6. The effect of DLC/a-Si thickness ratios on the $\mathrm{sp}^{3}$ content of DLC was analyzed by Raman spectroscopy. The results show that a-Si layer has no effect on the structure of DLC film. Furthermore, the upper shift in $G$ wavenumber and the decrease in $I_{D} / I_{G}$ inform that $\mathrm{sp}^{3}$ content of the film is directly proportional to DLC thickness. The plot modified from the three-stage model informed that the structural characteristics of DLC/a-Si bilayer films are located close to the tetrahedral amorphous carbon. This information may be important for analyzing and developing bilayer protective films for future hard disk drive.
\end{abstract}

\section{Introduction}

In current hard disk drive technology, data are written and retrieved by a magnetic recording head, which consists of a magnetic transducer and a sensor, flying over the disk surface at a height of $10 \mathrm{~nm}$ or lower. At this tiny space, problems may occur if the recording head contacts with the disk surface during operation. Thus, coating recording head and disk surface with a protective film is highly required as it will minimize the contact force at the head/disk interface. At present, DLC film is becoming a preferred coating for the head and disk surface because of their unique properties of the $\mathrm{sp}^{3}$ structure that is similar to the physical properties of diamond, such as high density, high wear resistance, low friction coefficient, chemical inertness, and optical transparency [1-6].

DLC film can be prepared by various methods, for example, ion beam assisted deposition [8], sputtering [9], filtered cathodic arc [10], plasma-assisted chemical vapor deposition [11], and pulsed laser deposition [12]. However, different preparation methods may offer DLC film of different forms, such as hydrogenated form of amorphous carbon (a-C:H) and nonhydrogenated form called tetrahedral amorphous carbon (ta-C). Most of the a-C:H form contains $\mathrm{sp}^{3}$ fractions of less than $50 \%$, while the ta-C can contain $\mathrm{sp}^{3}$ fractions of up to $85 \%[13,14]$. Thus, the preferred structure of DLC for being a protective film is the tetrahedral amorphous carbon (ta-C). Liu and Wang [3], and Liu et al. [15] reported the coating of Si layer with a thickness of $1 \mathrm{~nm}$ on the substrate as seed layer before coating DLC film. However, the effects of Si layer on the $\mathrm{sp}^{3}$ content of DLC film were not discussed.

In this work, amorphous silicon (a-Si) layer was initially deposited on germanium $(\mathrm{Ge})$ substrate using direct current (DC) magnetron sputtering. Diamond-like carbon (DLC) layer was then deposited on the a-Si layer using pulsed filter cathodic arc (PFCA). The effects of a-Si layer thickness on the $\mathrm{sp}^{3}$ content of DLC film were studied by varying the thicknesses of a-Si and DLC layers from 2 to $6 \mathrm{~nm}$ and 2 to $9 \mathrm{~nm}$, respectively, depending on the DLC/aSi thickness ratios, including $2 / 2,2 / 6,4 / 4,6 / 2$, and $9 / 6$. Therefore, the total thicknesses of DLC/a-Si films are $4,8,8$, 8 , and $15 \mathrm{~nm}$, respectively. The $\mathrm{sp}^{3}$ content of DLC/a-Si films 
were characterized by Raman spectroscopy and transmission electron microscopy (TEM).

\section{Experimental}

DLC/a-Si bilayer films were deposited using the pulsed filtered cathodic arc (PFCA) system. The system consists of four vacuum chambers, including (i) load lock chamber, (ii) transfer chamber, (iii) preclean chamber, and (iv) pulsed filtered cathodic arc (PFCA) chamber. Crystalline n-type germanium wafers with (100) orientation, and a dimension of $1 \times 1 \mathrm{~cm}^{2}$ with a thickness of $0.05 \mathrm{~cm}$ were used as a substrate. The DLC/a-Si deposition process consists of three steps: (i) the substrate was cleaned with low energy $\mathrm{Ar}^{+}$ion plasma etching at an incident angle of $60^{\circ}$ with respect to substrate, for $60 \mathrm{sec}$, (ii) a-Si seed layer was deposited on the Ge substrate at an incident angle of $44^{\circ}$ with respect to the substrate, using DC magnetron with a power of $150 \mathrm{~W}$ and an argon gas flow rate of $40 \mathrm{sccm}$, and (iii) DLC layer was deposited at the normal incident angle with respect to the substrate using pulsed filtered cathodic arc (PFCA) with a pulse frequency of $1 \mathrm{~Hz}$, arc voltage of $950 \mathrm{~V}$, and coil voltage of $900 \mathrm{~V}$. The first two steps were carried out in preclean chamber, while the third step was prepared in the PFCA chamber. The film thickness was monitored using in situ ellipsometry. Table 1 shows the details of the DLC/a-Si thickness ratios for all DLC/a-Si films.

Thickness of DLC/a-Si film on Ge substrate was investigated using TEM (Tecnai G2 20). It should be noted that before carrying out TEM cross-sectional image, the DLC/aSi film was coated with $\mathrm{Cr}$ layer. This additional layer acts as a protective layer to prevent passivation from ion bombardment during cross-sectional preparation using a focused ion beam (FIB). Raman measurements were performed with a Renishaw inVia Reflex Raman Spectrometer at $514 \mathrm{~nm}$ of $\mathrm{Ar}^{+}$ion gas laser. The laser output power of $20 \mathrm{~mW}$ and $50 \mathrm{x}$ objective lens were used, which resulted in an incident power at the sample of approximately $4 \mathrm{~mW}$. The scan range was from 1180 to $1800 \mathrm{~cm}^{-1}$. The raw spectra were fitted using Gaussian profile to obtain smooth curve. Then, the smooth curve was fitted with two Gaussian-Lorentzian functions corresponding to the $G$ and $D$ band wavenumbers. $G$ band is originated from the stretching vibration of any pair of $\mathrm{sp}^{2}$ sites, whether in $\mathrm{C}=\mathrm{C}$ chains or in hexagonal rings, while $D$ band is the breathing mode of those $\mathrm{sp}^{2}$ sites only in rings, not in chains $[7,14]$.

\section{Results and Discussion}

Cross-sectional morphologies of DLC/a-Si films prepared from different DLC/a-Si thickness ratios $(2 / 2,2 / 6,4 / 4,6 / 2$, and 9/6) are similar, but they are different in the thickness of a-Si and DLC layers. Figure 1(a) shows a typical crosssectional TEM image of the bilayer film prepared from the DLC/a-Si thickness ratio of $9 / 6$ on Ge substrate. There are four regions presented in the image, including Ge substrate, a-Si layer, DLC layer, and Cr layer (a protective layer for preventing passivation from ion bombardment during
TABLE 1: Thickness of DLC and a-Si layers, DLC/a-Si thickness ratio, and total thickness of DLC/a-Si film.

\begin{tabular}{lccc}
\hline $\begin{array}{l}\text { DLC layer } \\
(\mathrm{nm})\end{array}$ & $\begin{array}{c}\text { Thickness } \\
\text { a-Si layer } \\
(\mathrm{nm})\end{array}$ & $\begin{array}{c}\text { DLC/a-Si } \\
\text { Thickness } \\
\text { ratio }\end{array}$ & $\begin{array}{c}\text { Total thickness } \\
(\mathrm{nm})\end{array}$ \\
\hline 2 & 2 & $2 / 2$ & 4 \\
2 & 6 & $2 / 6$ & 8 \\
4 & 4 & $4 / 4$ & 8 \\
6 & 2 & $6 / 2$ & 8 \\
9 & 6 & $9 / 6$ & 15 \\
\hline
\end{tabular}

TABLE 2: Information extracted from Raman spectra of DLC/a-Si films prepared from different DLC/a-Si thickness ratios.

\begin{tabular}{lccc}
\hline $\begin{array}{l}\text { DLC/a-Si } \\
\text { thickness ratio }\end{array}$ & $\begin{array}{c}\text { Total } \\
\text { thickness }(\mathrm{nm})\end{array}$ & $\begin{array}{c}G \\
\text { Wavenumber }\left(\mathrm{cm}^{-1}\right)\end{array}$ & $I_{D} / I_{G}$ \\
\hline $2 / 2$ & 4 & 1552 & 1.528 \\
$2 / 6$ & 8 & 1552 & 1.507 \\
$4 / 4$ & 8 & 1557 & 0.744 \\
$6 / 2$ & 8 & 1561 & 0.568 \\
$9 / 6$ & 15 & 1565 & 0.453 \\
\hline
\end{tabular}

preparation of cross-sectional film). Two regions according to a-Si and DLC layers are of our interest. The morphology and density of a-Si and DLC layers are slightly different as shown in white and gray and are also seen in Figure 1(b) which is the cropped image of Figure 1(a) after brightness and contrast adjustment.

The structural characteristics of DLC layer prepared from different DLC/a-Si thickness ratios were investigated by Raman spectroscopy. Figure 2 shows Raman spectra of DLC/a-Si films prepared from the DLC/a-Si thickness ratios of $2 / 2,2 / 6,4 / 4,6 / 2$, and $9 / 6$. The spectra exhibit a broad wavenumber ranging from 1200 to $1800 \mathrm{~cm}^{-1}$. This informs the formation of $\mathrm{sp}^{2}$ and $\mathrm{sp}^{3}$ contents, which can be used for indicating the structural characteristics of DLC layer [16].

To have further understanding on the structural characteristics of the bilayer film, Raman spectrum of DLC/aSi film from each thickness ratio was fitted to obtain $G^{-}$ band wavenumber and $\mathrm{D} / \mathrm{G}$ intensity ratio $\left(I_{D} / I_{G}\right)$ using Gaussian function. The typical fitted spectrum of DLC/aSi film is shown in Figure 3 which includes graphite $(G)$ band, disorder $(D)$ band, and fitted spectrum. G-band wavenumber and $I_{D} / I_{G}$ ratio from the fitted spectra are given in Table 2. For DLC/a-Si ratios of $2 / 2$ and $2 / 6$, where DLC film thickness is $2 \mathrm{~nm}$ and a-Si layer thicknesses are 2 and $6 \mathrm{~nm}$, respectively. The $G$ band positions for these two ratios are the same with a $G$ wavenumber of $1552 \mathrm{~cm}^{-1}$. This result indicates that a-Si layer has no effect on the structure of taC film. For DLC/a-Si ratios of $4 / 4,6 / 2$, and $9 / 6$, that is the thicknesses of DLC film are 4, 6, and $9 \mathrm{~nm}$, respectively. It is seen from Table 2 that the $G$ band position shifts to the higher wavenumber when the thickness of DLC film increases. This result implies that the structure of ta-C film depends only on the thickness of DLC film. The thicker DLC layer in the bilayer film offers higher vibrational energy which comes 


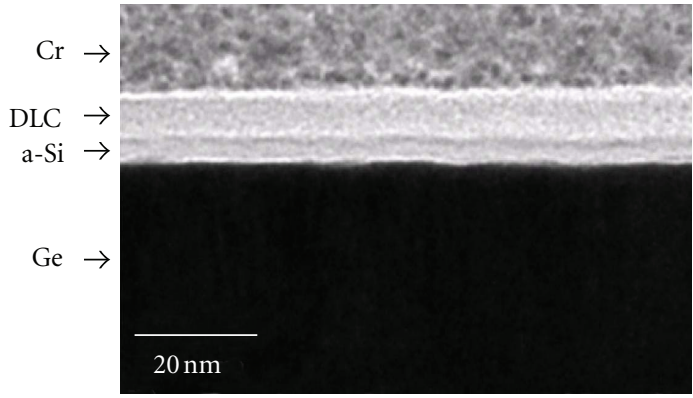

(a)

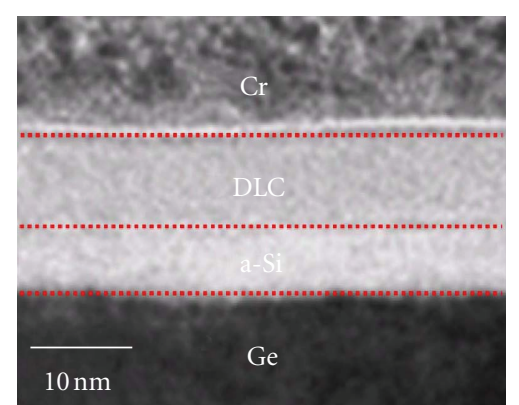

(b)

Figure 1: (a) Cross-sectional TEM image of the bilayer film prepared from the DLC/a-Si thickness ratio of 9/6. (b) Cropped image from (a) with a brightness and contrast adjustment to provide a clear vision on the cross-sectional morphology of the films.

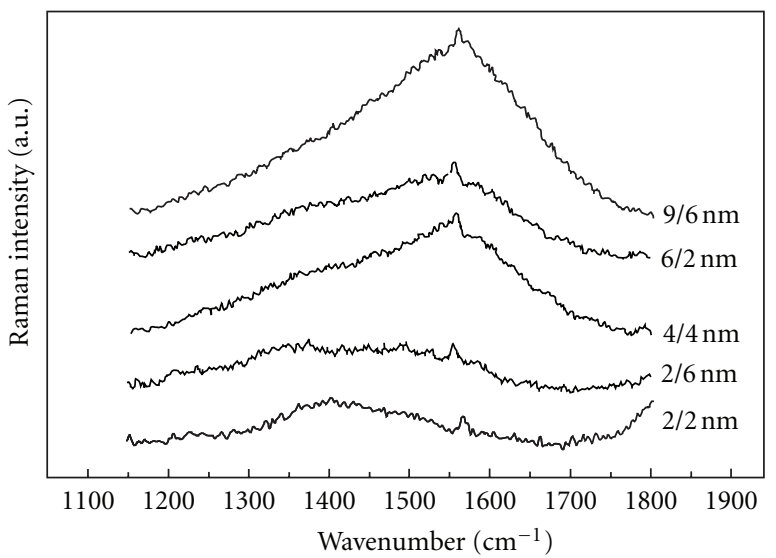

Figure 2: Raman spectra of the bilayer films prepared from different DLC/a-Si thickness ratios: 2/2, 2/6, 4/4, 6/2, and 9/6.

from the change in bond stretching mechanism of any pair of $\mathrm{sp}^{2}$ sites due to the increase in short $\mathrm{C}=\mathrm{C}$ chain contents $[7,17]$ and result in the higher $\mathrm{sp}^{3}$ contents of ta-C structure $[14,18,19]$.

This result is also in agreement with the three-stage model reported by Ferrari and Robertson [7] to analyze Raman spectra of ta-C film. As shown in Figure 4, the relationship between $G$-band wavenumber of DLC/a-Si film and its thickness ratios was plotted. The data analyzed according to the three-stage model inform that $G$-band wavenumber can be used for indicating $\mathrm{sp}^{2}$ configuration of the material. For example, the $G$-band wavenumbers at $1580,1600,1510$, and $1570 \mathrm{~cm}^{-1}$ indicate the $\mathrm{sp}^{2}$ site from graphite, nanocrystalline-graphite (NC-graphite), amorphous carbon (a-C), and tetrahedral amorphous carbon (ta-C), respectively. Thus, Figure 4 confirms that the structural characteristics of DLC layers prepared from all types of DLC/a-Si thickness ratio are very close to the tetrahedral amorphous carbon $(\mathrm{ta}-\mathrm{C})$ structure since they are located close to the ta-C structure.

To confirm the previous explanation on the $\mathrm{sp}^{3}$ content, the $I_{D} / I_{G}$ ratio which is normally correlated to the $\mathrm{sp}^{3} / \mathrm{sp}^{2}$ fraction was evaluated. Ideally, the $I_{D} / I_{G}$ value for ta-C should be close to zero since the $D$ band is the breathing

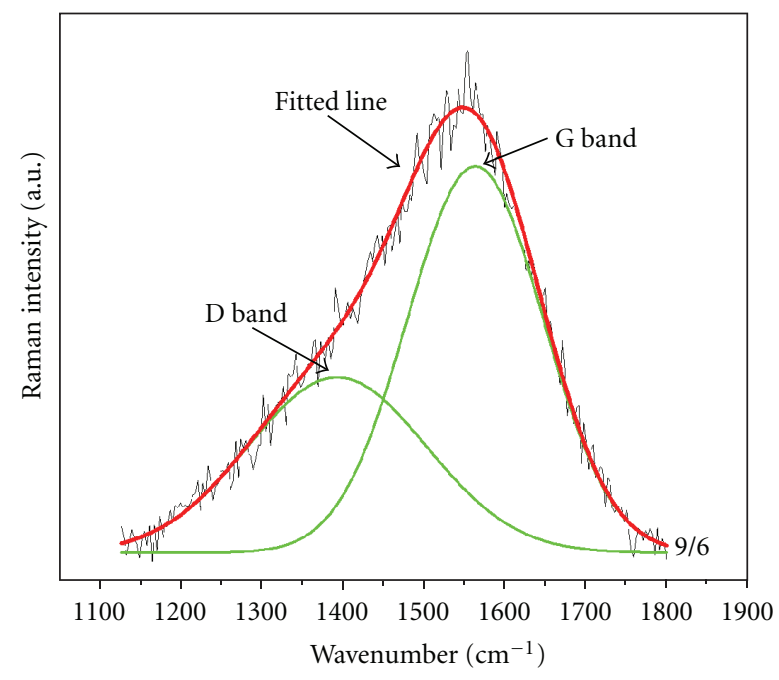

FIGURE 3: Raman spectrum of the bilayer film was fitted into $G$ and $D$ bands. The film was prepared from the DLC/a-Si thickness ratio of $9 / 6$.

mode of $\mathrm{sp}^{2}$ sites only in rings, not in chains [7]. The $I_{D} / I_{G}$ ratios of DLC/a-Si films are $1.528,0.568$, and 0.453 for the DLC/a-Si thickness ratios of $2 / 2,6 / 2$, and $9 / 6$, respectively, as shown in Figure 5. This result indicates that when DLC layer becomes thicker, there are more $\mathrm{sp}^{3}$ contents. Moreover, from the plot in Figures 4, and 5, it was found that the thickness of a-Si layer has no effect on the DLC formation and the $\mathrm{sp}^{3} / \mathrm{sp}^{2}$ fraction. However, it may has an important role in the mechanical properties of the DLC film such as adhesion, hardness, and wear resistance which will be necessary for future investigation.

\section{Conclusions}

DLC/a-Si bilayer films of different DLC/a-Si thickness ratios were deposited on Ge substrates. $G$-band wavenumber shifts to higher value when the thickness of DLC layer increases due to higher $\mathrm{sp}^{3}$ contents. This is also confirmed by the decrease in $I_{D} / I_{G}$ ratio. The plot modified from the three-stage model points out that the structural characteristics of DLC layers 


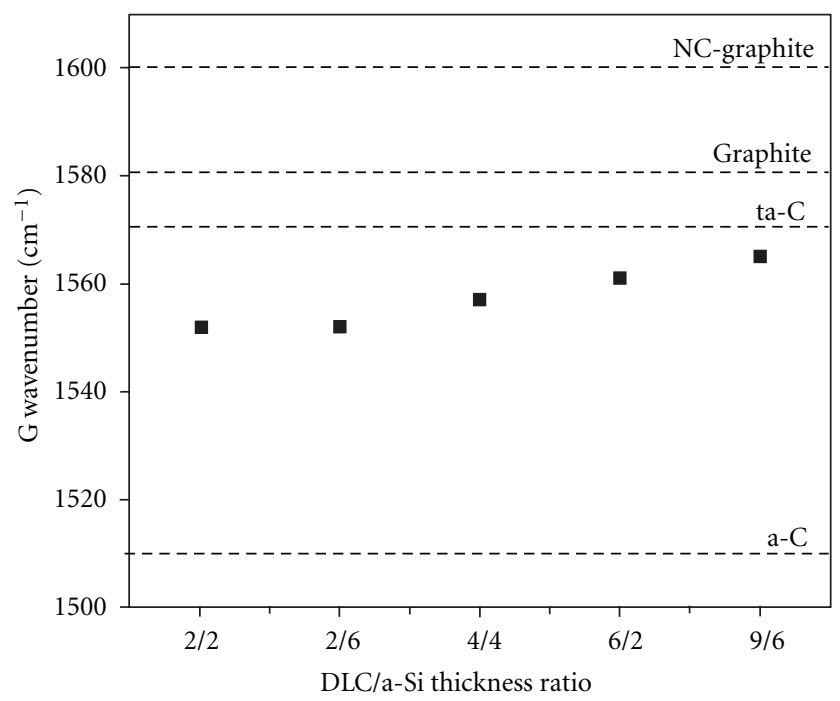

FIgure 4: The plot modified from the three-stage model [7] to obtain structural characteristics of DLC in the bilayer film. Four structures are presented: nanocrystalline-graphite (NC-graphite), graphite, amorphous carbon (a-C), and tetrahedral amorphous carbon (ta-C).

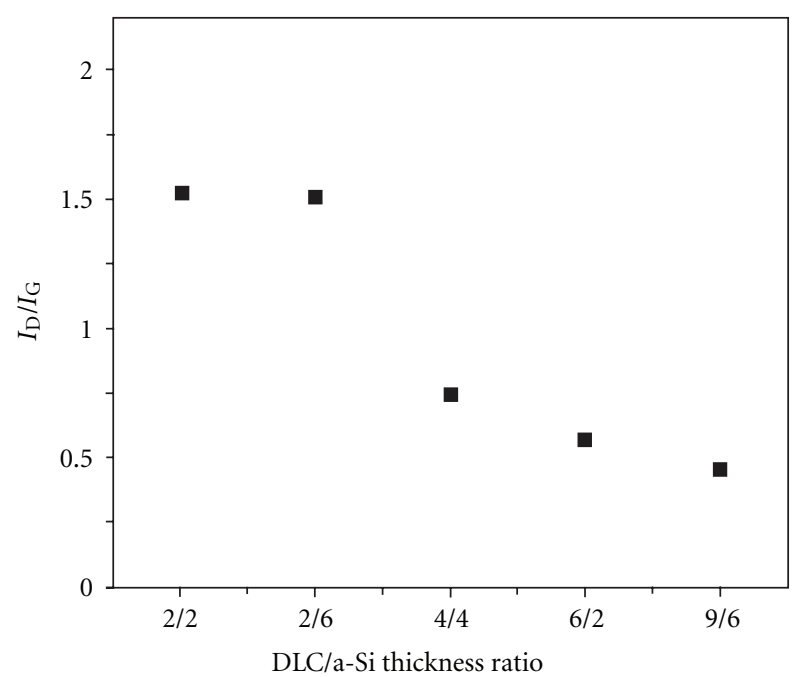

Figure 5: Plot of $I_{D} / I_{G}$ ratio of the bilayer film as a function of DLC/a-Si thickness ratio. Decrease in the $I_{D} / I_{G}$ ratio represents the increase in $\mathrm{sp}^{3}$ contents of the films.

prepared from all types of DLC/a-Si thickness ratio are very close to the tetrahedral amorphous carbon (ta-C). Thickness of a-Si layer, a seed layer to improve the adhesion between DLC and substrate, barely affects the DLC formation and the $\mathrm{sp}^{3}$ bonded. This information can be applied for developing DLC for bi- and multilayer protective coatings in which structural characteristics such as $\mathrm{sp}^{3}$ content can be analyzed easily from the modified plot.

\section{Acknowledgments}

This work was supported by Industry/University Cooperative Research Center (I/UCRC) in HDD Advanced Manufacturing, Institute of Field roBOtics, King Mongkut's University of Technology Thonburi and National Electronics and Computer Technology Center, National Science and Technology Development Agency. It was also supported by KMUTT under the National Research University Project and Thailand Center of Excellence in Physics.

\section{References}

[1] J. Robertson, "Ultrathin carbon coatings for magnetic storage technology," Thin Solid Films, vol. 383, no. 1-2, pp. 81-88, 2001.

[2] J. Robertson, "Diamond-Like Amorphous carbon," Materials Science and Engineering, vol. 37, pp. 129-281, 2002.

[3] F. X. Liu and Z. L. Wang, "Thickness dependence of the structure of diamond-like carbon films by Raman spectroscopy," Surface and Coatings Technology, vol. 203, no. 13, pp. 1829$1832,2009$.

[4] J. J. Rha, S. C. Kwon, J. R. Cho, S. Yim, and N. Saka, “Creation of ultra-low friction and wear surfaces for micro-devices using carbon films," Wear, vol. 259, no. 1-6, pp. 765-770, 2005.

[5] H. Kohira, V. Prabhakaran, and F. E. Talke, "Effect of air bearing design on wear of diamond-like carbon coated proximity recording sliders," Tribology International, vol. 33, no. 5, pp. 315-321, 2000.

[6] N. Gopinathan, C. Robinson, and F. Ryan, "Characterization and properties of diamond-like carbon films for magnetic recording application," Thin Solid Films, vol. 355, pp. 401-405, 1999.

[7] A. C. Ferrari and J. Robertson, "Interpretation of Raman spectra of disordered and amorphous carbon," Physical Review B, vol. 61, no. 20, pp. 14095-14107, 2000.

[8] C. Weissmantel, K. Bewilogua, D. Dietrich et al., "Structure and properties of quasi-amorphous films prepared by ion beam techniques," Thin Solid Films, vol. 72, no. 1, pp. 19-31, 1980.

[9] N. Savvides, "Optical constants and associated functions of metastable diamondlike amorphous carbon films in the energy range 0.5-7.3 eV," Journal of Applied Physics, vol. 59, no. 12, pp. 4133-4145, 1986.

[10] M. S. Leu, S. Y. Chen, J. J. Chang, L. G. Chao, and W. Lin, "Diamond-like coatings prepared by the filtered cathodic arc technique for minting application," Surface and Coatings Technology, vol. 177-178, pp. 566-572, 2004.

[11] J. Y. Shim, E. J. Chi, H. K. Baik, and S. M. Lee, "Structural, optical, and field emission properties of hydrogenated amorphous carbon films grown by helical resonator plasma enhanced chemical vapor deposition," Japanese Journal of Applied Physics, vol. 37, no. 2, pp. 440-444, 1998.

[12] H. J. Scheibe, D. Drescher, B. Schultrich, M. Falz, G. Leonhardt, and R. Wilberg, "The laser-arc: a new industrial technology for effective deposition of hard amorphous carbon films," Surface and Coatings Technology, vol. 85, no. 3, pp. 209214, 1996.

[13] A. Grill, "Diamond-like carbon: state of the art," Diamond and Related Materials, vol. 8, no. 2-5, pp. 428-434, 1999.

[14] M. V. Gradowski, A. C. Ferrari, R. Ohr et al., "Resonant Raman characterisation of ultra-thin nano-protective carbon layers 
for magnetic storage devices," Surface and Coatings Technology, vol. 174-175, pp. 246-252, 2003.

[15] F. X. Liu, K. L. Yao, and Z. L. Liu, "Substrate bias effect on structure of tetrahedral amorphous carbon films by Raman spectroscopy," Diamond and Related Materials, vol. 16, no. 9, pp. 1746-1751, 2007.

[16] P. J. Fallon, V. S. Veerasamy, C. A. Davis et al., "Properties of filtered-ion-beam-deposited diamondlike carbon as a function of ion energy," Physical Review B, vol. 48, no. 7, pp. 47774782, 1993.

[17] F. X. Liu, K. L. Yao, and Z. L. Liu, "Different substrate materials effect on structure of ta-C films by Raman spectroscopy for magnetic recording sliders," Journal of Non-Crystalline Solids, vol. 353, no. 26, pp. 2545-2549, 2007.

[18] K. W. R. Gilkes, S. Prawer, K. W. Nugent et al., "Direct quantitative detection of the sp3 bonding in diamond-like carbon films using ultraviolet and visible Raman spectroscopy," Journal of Applied Physics, vol. 87, no. 10, pp. 7283-7289, 2000.

[19] A. Grill, B. S. Meyerson, V. V. Patel, J. A. Reimer, and M. A. Petrich, "Inhomogeneous carbon bonding in hydrogenated amorphous carbon films," Journal of Applied Physics, vol. 61, no. 8 , pp. $2874-2877,1987$. 

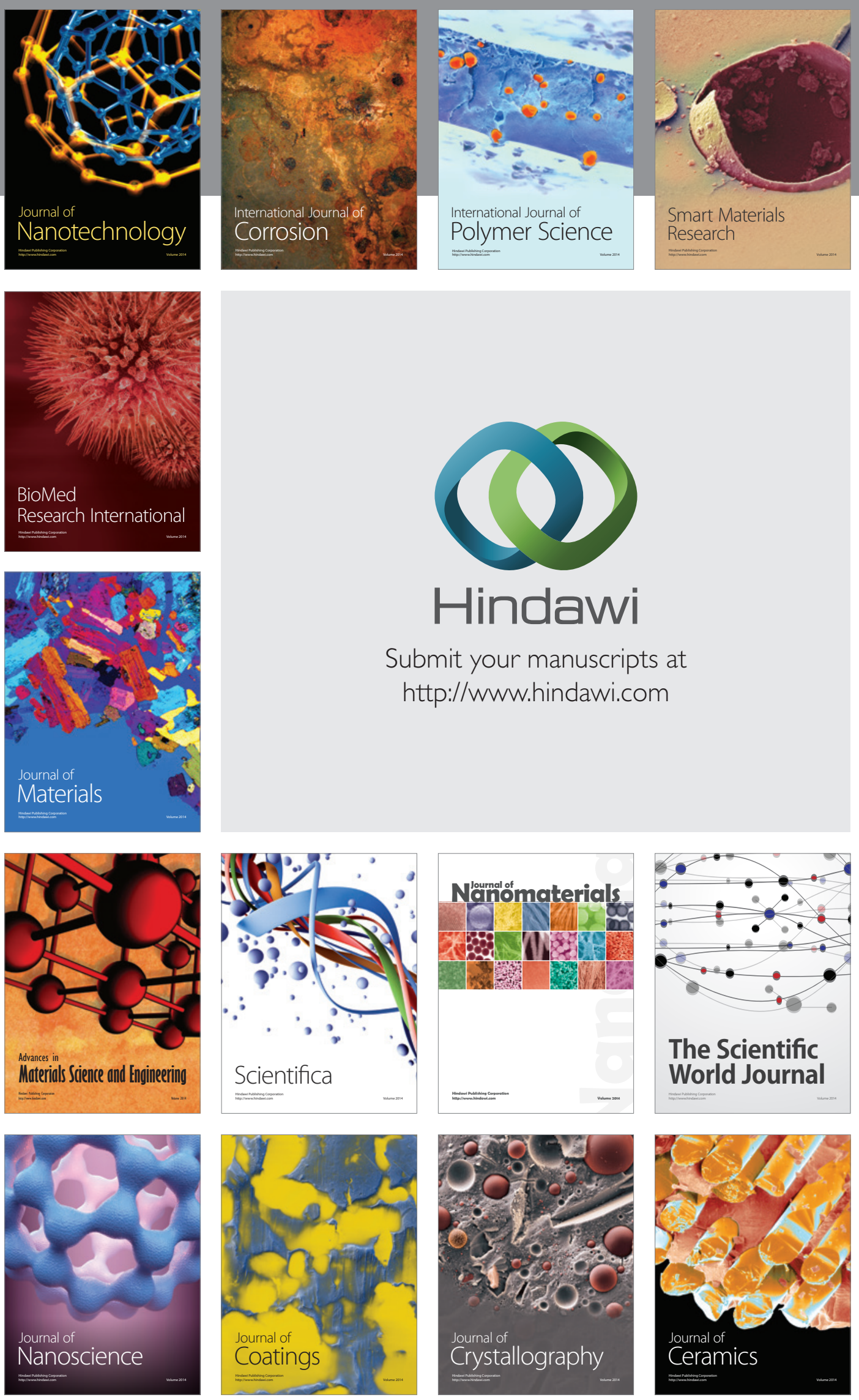

The Scientific World Journal

Submit your manuscripts at

http://www.hindawi.com

\section{World Journal}

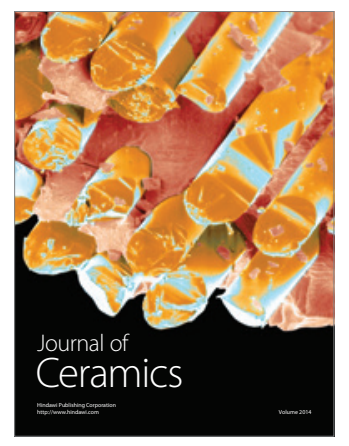

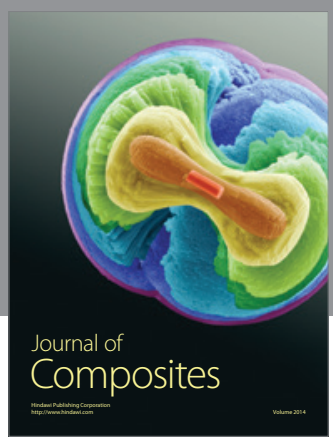
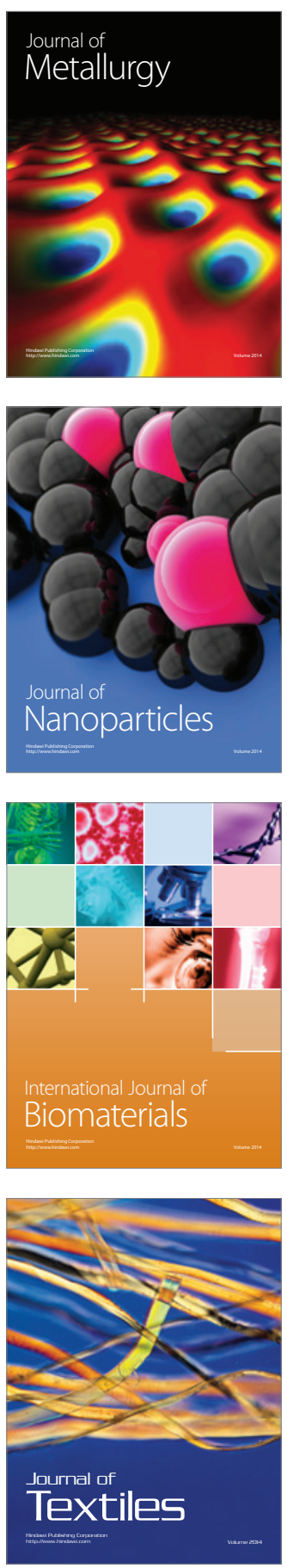